\title{
Subterahertz hypersound attenuation in silica glass studied via picosecond acoustics
}

\author{
S. Ayrinhac, ${ }^{1}$ M. Foret, ${ }^{2}$ A. Devos,${ }^{1}$ B. Rufflé, ${ }^{2}$ E. Courtens, ${ }^{2}$ and R. Vacher ${ }^{2}$ \\ ${ }^{1}$ Institut d'Électronique, de Microélectronique et de Nanotechnologie, UMR CNRS 8250 BP 69, \\ Avenue Poincaré, F-59652 Villeneuve d'Ascq Cedex, France \\ ${ }^{2}$ Laboratoire des Colloïdes, Verres et Nanomatériaux, UMR 5587 CNRS Université Montpellier II, F-34095 Montpellier Cedex 5, France
}

(Received 25 June 2010; revised manuscript received 17 September 2010; published 20 January 2011)

\begin{abstract}
We report picosecond acoustic measurements in silica-glass films grown by wet thermal oxidation on a (111) silicon substrate. The longitudinal acoustic phonons are observed over the range from 150 to $300 \mathrm{GHz}$ using an infrared pump and a second harmonic blue probe. The transducer is an aluminum thin film deposited on top. Multiple interference effects are analyzed and fully taken into account. They lead to a signal presenting rapid oscillations as a function of the sample thicknesses. The latter are determined by separate interferometry. Our remarkably precise acoustic attenuation results are found to follow rather well a model combining thermally activated relaxations and anharmonicity. New values for the optical absorption of silicon in the 400-to-500-nm region of the spectrum are obtained as a by-product.
\end{abstract}

DOI: 10.1103/PhysRevB.83.014204

\section{INTRODUCTION}

Sound attenuation in glasses exhibits a rich variety of phenomena depending on the frequencies and temperatures where it is observed. Results are available from $\sim 20 \mathrm{mK}$ to well over $1000 \mathrm{~K}$. At very low temperatures, the acoustic attenuation is dominated by the coupling of strain to tunneling within two-level systems. ${ }^{1-3}$ At intermediate temperatures, typically above $10 \mathrm{~K}$, the thermally activated relaxation (TAR) of defects which can be modeled by two-well potentials becomes the major source of attenuation. ${ }^{4-6}$ At still higher temperatures, the anharmonic interaction of acoustic waves with the thermal phonon bath becomes important. ${ }^{7,8}$ This initially varies with the square of the frequency, making it the dominant source of attenuation at high frequencies, typically above $10 \mathrm{GHz}$. The evolution of hypersound attenuation at still higher frequencies remains currently debated. Around $1 \mathrm{THz}$, an attenuation proportional to the fourth power of the frequency was observed in several glasses. ${ }^{9-12}$ It can be explained by resonance with the low-frequency wing of additional vibrational modes forming the boson peak. ${ }^{13}$ An alternate model is that pure elastic disorder becomes relevant at the short length scale corresponding to the acoustic wavelength. ${ }^{14,15}$ To clarify such issues, investigations at frequencies between $\sim 100 \mathrm{GHz}$ and $\sim 1 \mathrm{THz}$ would be very valuable. This unfortunately falls into a gap where scattering techniques cannot be used for the investigation of acoustic propagation and attenuation.

Spectroscopic techniques for the study of sound now cover a broad range of frequencies, from sub- $\mathrm{Hz}$ to tens of $\mathrm{GHz}$. The higher frequencies are generally observed with Brillouin light scattering (BLS). The upper range can be increased using the backscattering geometry together with UV excitation. ${ }^{16,17}$ There is, however, a limit set by the optical absorption edge of the sample. ${ }^{18}$ It corresponds to acoustic waves around $100 \mathrm{GHz}$ in the case of vitreous silica. The samples remain then opaque to electromagnetic (EM) radiation up to soft x-rays. Using near forward $\mathrm{x}$-ray scattering with radiation around $20 \mathrm{keV}$ allows observing acousticlike excitation at frequencies down to about $1 \mathrm{THz} .{ }^{19}$ It is this inelastic $\mathrm{x}$-ray scattering (IXS) technique that was used in Refs. 9-12. It is thus optical absorption which is responsible for the scattering-measurement gap between $\sim 100 \mathrm{GHz}$ and $\sim 1 \mathrm{THz}$.

Picosecond optical techniques (POT) now offer a possible approach to access acoustic properties in the region of that gap. The general idea is to generate a hypersound pulse by the absorption of a femtosecond optical pulse, the pump, and to follow its evolution by a second optical pulse, the probe, delayed in time. ${ }^{20-23}$ One approach consists in probing the time of flight of an acoustic pulse bouncing back and forth in a thin film. This produces echoes whose separation depends on the acoustic velocity. An analysis of the echo shape allows extracting information on the acoustic attenuation as a function of frequency. ${ }^{22,24}$ Another approach is to use the oscillations produced by the interference of the probe partly reflected at the sample surface with its reflection by the moving acoustic pulse. The latter can be viewed as a superposition of space-time Fourier components. For momentum conservation reasons the light interacts with one of these, and thus this approach is limited to the same frequency region accessible to Brillouin scattering. ${ }^{25}$ However, for a transparent dielectric film deposited on a substrate with significantly higher refractive index and acoustic velocity, the oscillations originating from the substrate correspond to appreciably higher frequency acoustic waves than these seen in the dielectric. It was recently shown that a study of these substrate oscillations as a function of the dielectric-film thickness $L$ allows determining hypersound attenuation in the dielectric at frequencies beyond these accessible to BLS. ${ }^{26,27}$ For silica films on a silicon substrate, the success of such experiments depends on using a probe pulse in the blue region of the spectrum, enhancing the acousto-optic coupling to the substrate. ${ }^{28}$

The present paper is entirely devoted to measurements of the acoustic absorption with the latter approach. Section II explains in simple terms the principle of the experiment. It exposes the method without technical details, reserved for subsequent sections. It also describes the samples used. Section III presents an analysis of the hypersound pulse and of its optical reflection coefficient. Section IV explains the multiple interferences and it shows how their effect can be 
checked by performing a study as a function of the probe carrier wavelength. Section V discusses the precise determination of the film thicknesses $L$ and of the required indices. Section VI is devoted to the new sound-attenuation measurements on vitreous silica, with full account for interferences. It also discusses former results obtained on differently prepared silica films. While the accuracy of the latter results is lower, the comparison indicates that silica films of different preparation and acoustic velocities can have rather similar acoustic absorption around $200 \mathrm{GHz}$. Section VII concludes the paper, including an outlook toward further possible developments. An appendix lists ancillary formulas for the calculation of interferences.

\section{PRINCIPLE OF THE METHOD, SAMPLES, AND TREATMENT OF THE RAW DATA}

The typical experimental arrangement is illustrated in Fig. 1. A femtosecond laser source generates the pump pulse, of duration $t_{\mathrm{pu}}$, and a time delayed, frequency-doubled probe pulse of duration $t_{\mathrm{pr}}$. The pump and probe are incident from the air $\{0\}$ on a sample formed of a thin metallic film $\{1\}$ of thickness $d^{\prime}=z_{1}$ deposited on a transparent dielectric $\{2\}$ of thickness $L=z_{2}-z_{1}$, all supported by a substrate $\{3\}$. Both pump and probe are nearly normal to the films so that the cosine of their inclination is practically equal to one. This inclination, in reality much smaller than sketched in Fig. 1, will be neglected in what follows. Also, the lateral extent of the optical beams is very large compared to $L$ so that only the dependence on $z$, the coordinate perpendicular to the films, will be consequent. In our implementation, the pump is a transform-limited pulse whose carrier is tunable in the near infrared with $t_{\mathrm{pu}} \simeq 120 \mathrm{fs}$, and $t_{\mathrm{pr}} \simeq t_{\mathrm{pu}}$. In these experiments, $\{1\}$ is aluminum, with $d^{\prime} \simeq 8 \mathrm{~nm}$ for our main sample series, $\{2\}$ is silica glass, and $\{3\}$ is a single crystal of silicon. Two series of samples have been used. For each series, different $L$ values up to $L \approx 1 \mu \mathrm{m}$ are available for experiments.

The pump produces at time $t=0$ a rapid increase in the metallic film temperature and thereby a compressive stress. This transforms into an acoustic wave that bounces within the thin metallic film. At the interface $\{1-2\}$, this immediately launches a strain pulse in the dielectric. Its typical duration

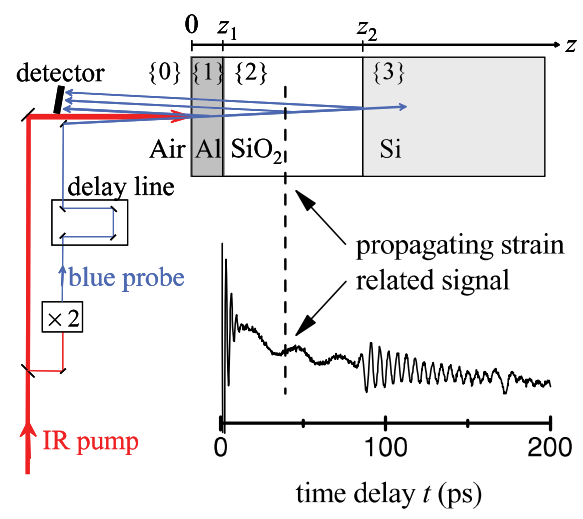

FIG. 1. (Color online) The experimental arrangement together with the coordinates and labels used for the various layers. A typical signal, whose details are analyzed in Fig. 2, is shown for illustration. is of the order of the acoustic round trip in the metallic layer $\{1\}, 2 \tau=2 d^{\prime} / v_{1}$, where $v_{1}$ is the acoustic velocity in the metal. The spatial extent $2 d$ of the strain pulse in $\{2\}$ is of the order of $2 \tau v_{2}$, where $v_{2}$ is the acoustic velocity in the dielectric. In our implementation, $2 \tau \simeq 3 \mathrm{ps}$, and since $v_{2} \simeq v_{1}$, the spatial extent in $\{2\}$ is $2 d \simeq 15 \mathrm{~nm}$. At time $t$, this strain is located at depth $Z(t)=z_{1}+v_{2} t$. An experimental run consists in observing, as a function of the delay time $t$, the reflection of the probe on the traveling strain pulse interfering with all other reflections from the layered sample. It should be noted that $t_{\mathrm{pr}}$ is so short that the traveling strain pulse is nearly stationary for that duration, as it only moves by $t_{\mathrm{pr}} v_{2} \simeq 0.7 \mathrm{~nm}$, which is small compared to $2 d$. On the other hand, $t_{\mathrm{pr}}$ is sufficiently long to cover a great many times the thickness $L$ of the dielectric since $t_{\mathrm{pr}} c / n_{2} \simeq 25 \mu \mathrm{m}$, where $c$ is the velocity of light in vacuum and $n_{2}$ the refractive index in medium $\{2\}$.

The raw data obtained in a typical run are illustrated in Fig. 1. The initial wiggles up to $t \approx 10 \mathrm{ps}$ are produced by the acoustic wave bouncing in the metallic film. They are superposed to an oscillatory signal of longer period $T_{2}$ and amplitude $A_{2}$ produced by the strain in $\{2\}$. It results from the interference of the reflection of the probe on the moving strain pulse with all other reflections from the sample. The oscillation period corresponds to an increase of $Z$ by $\Delta Z$ such that the round-trip phase shift $2 k_{2} Z$ is augmented by $2 \pi$. Here $k_{2}$ is the wave vector of the probe light in $\{2\}, k_{2}=2 \pi n_{2} / \lambda_{0}$, where $\lambda_{0}$ is the probe carrier wavelength in vacuum. Therefore,

$$
T_{2}=\frac{\Delta Z}{v_{2}}=\frac{\lambda_{0}}{2 n_{2} v_{2}}=\left(v_{2}^{\mathrm{ac}}\right)^{-1} .
$$

This is equivalent to the Bragg condition. It corresponds to the backscattering of the EM-wave on the Fourier component at frequency $v_{2}^{\mathrm{ac}}$ in the acoustic wave packet. In other words, the strength of the signal $A_{2}$ is proportional to that Fourier amplitude. Strictly speaking, the frequency of the back-scattered EM wave is downshifted by the Brillouin shift $v_{2}^{\text {ac }}$. In the present case, that shift is not significant compared to the relatively enormous frequency spread of the incoming EM pulse.

At sufficiently long delays, for $t>t_{2}=L / v_{2}$, the strain wave has reached the interface $\{2-3\}$, where it is partly reflected and partly transmitted. The reflected part produces a component to the signal of the same period $T_{2}$ and of reduced amplitude $A_{2}^{\prime}$. Here, $A_{2}^{\prime} \approx 0.25 A_{2}$. This is superposed to the interferences produced by the transmitted strain pulse propagating in $\{3\}$. For similar phase-shift reasons as mentioned earlier, these lead to a signal of period $T_{3}$ given by

$$
T_{3}=\frac{\lambda_{0}}{2 n_{3} v_{3}}=\left(v_{3}^{\mathrm{ac}}\right)^{-1} \text {. }
$$

Here $n_{3}$ and $v_{3}$ correspond to medium $\{3\}$. For a silica film on a silicon substrate, $v_{3}^{\text {ac }}$ typically equals 5-6 times $v_{2}^{\text {ac }}$ depending on the dispersion of $n_{2}$ and $n_{3}$ with $\lambda_{0}$ and on the orientation of the silicon crystal that determines $v_{3}$. The strength of the signal of period $T_{3}$ is proportional to the amplitude of the Fourier component at frequency $v_{3}^{\text {ac }}$ contained in the acoustic pulse in medium $\{3\}$. One observes in Fig. 1 that this oscillatory signal is damped. This decay is not due to the acoustic attenuation in $\{3\}$, as the latter is very small in silicon, ${ }^{29}$ but to the absorption of the probe light by the semiconductor. It multiplies the 
oscillations of period $T_{3}$ by a decaying exponential of time constant

$$
\tau_{3}=\frac{\lambda_{0}}{4 \pi \kappa_{3} v_{3}},
$$

where $\kappa_{3}$ is the imaginary part of the complex index $\tilde{n}_{3}=$ $n_{3}+i \kappa_{3}$. However, the acoustic absorption at frequency $v_{3}^{\text {ac }}$ in the dielectric of thickness $L$ affects the initial amplitude $A_{3}$ of the signal originating from the substrate at $z_{2}$. The acoustic attenuation is given by $\exp \left[-\alpha_{\mathrm{ac}}\left(v_{3}^{\mathrm{ac}}\right) L\right]$, where $\alpha_{\mathrm{ac}}$ is the frequency-dependent amplitude absorption coefficient in medium $\{2\}$. An investigation of $A_{3}$ as a function of $L$ thus provides a means of studying hypersound attenuation in the film at the much higher frequency $v_{3}^{\text {ac }} \gg v_{2}^{\text {ac }}$, not accessible to ordinary Brillouin scattering. It is convenient to normalize $A_{3}$ by the amplitude $A_{2}$ as this eliminates such effects as fluctuations in the laser intensity. In the present case the attenuation over the length $L$ at frequency $\nu_{2}^{\text {ac }}$ is negligible, so that this provides a very convenient normalization. The observed ratio $A_{3} / A_{2}$ strongly depends on multiple reflections within the structure. The effect of the latter can be condensed into a multiplicative coefficient $f_{3} / f_{2}$ that will be called the sensitivity ratio. A measurement of the amplitude ratio $A_{3} / A_{2}$ as a function of $L$, with due account for the sensitivity ratio, thus provides information on the acoustic attenuation at frequency $v_{3}^{\text {ac }}$. This is the principle of the present measurement.

Our main series of five samples, designated here by wetox, was prepared by wet thermal oxidation of silicon. The substrates are cut from a (111) silicon wafer. This orientation gives the highest velocity $v_{3} \simeq 9360 \mathrm{~m} / \mathrm{s}$ and thus the highest achievable frequency $v_{3}^{\text {ac }}$ according to Eq. (2). The oxidation was performed at a substrate temperature of $1050{ }^{\circ} \mathrm{C}$ in a mixed gas of $\mathrm{O}_{2}$ and $\mathrm{H}_{2}$ flowing at the rate of 1.5 and 2.5 standard $\mathrm{L} / \mathrm{min}$, respectively. The nominal oxide thicknesses range from 300 to $1000 \mathrm{~nm}$. Using in Eq. (1) the average period $T_{2}$ obtained from a very large number of measurements, the product $n_{2} v_{2}$ is determined. Assuming the bulk value for $n_{2}$, we find then $v_{2}=5995 \pm$ $10 \mathrm{~m} / \mathrm{s}$. This value of the longitudinal sound velocity agrees with that of good quality bulk silica, $v_{2}=5980 \mathrm{~m} / \mathrm{s}$. The five samples were aluminized simultaneously with a nominally $10-\mathrm{nm}$ film. It was prepared by electron-beam evaporation at the rate of $0.1 \mathrm{~nm} / \mathrm{s}$ under a base pressure below $10^{-7}$ Torr. The effective Al thickness was determined optically and found to be $8 \mathrm{~nm}$, as explained in Sec. V. In a second series of four samples, designated as LPCVD, the oxide layers were prepared by low-pressure chemical vapor deposition at the ST Microelectronics facility in Crolles, France. These four samples had also been aluminized in our laboratory. Experiments on that series have already been reported in Ref. 27. For that series the substrate has a (100) orientation and the four samples have nominal thicknesses from 300 to $1200 \mathrm{~nm}$ in 300-nm steps. The silica was deposited from a silane-oxygen mixture on the substrate maintained at $\sim 700^{\circ} \mathrm{C}$. With the same assumption concerning $n_{2}$, we find from $T_{2}$ measurements that $v_{2}=5912 \pm 10 \mathrm{~m} / \mathrm{s}$ for the LPCVD samples, a value which is a little small compared to bulk silica. In this regard it can be mentioned that we also found $v_{2} \simeq 5660 \mathrm{~m} / \mathrm{s}$ for samples obtained by plasma-enhanced $\mathrm{CVD}$ on substrates maintained at $350^{\circ} \mathrm{C}$. That value of $v_{2}$

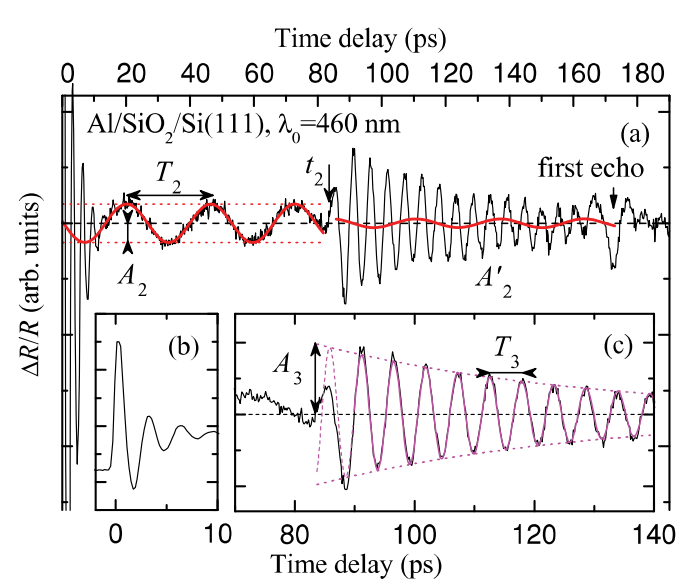

FIG. 2. (Color online) (a) Structure of the signal observed on the wetox sample of $503 \mathrm{~nm}$ thickness as a function of the probe delay. The first echo is produced by the acoustic pulse returning to the metallic layer; (b) the initial ringing; (c) the adjustment at $t \geqslant t_{2}$ to a decaying oscillation with the definition of the initial amplitude $A_{3}$ extrapolated to time $t_{2}$.

is really very small. Results will not be presented here on that third series of samples as we have no indication for the appropriate value of the refractive index $n_{2}$ in such a case.

To complete this discussion, Fig. 2 illustrates how the useful information is extracted from the raw data obtained in a single run. As seen in Fig. 1, the oscillatory signals are superposed to a decaying background. The latter is produced by the slow decrease of the metallic film temperature after the sudden heating by the pump. This slow decay depends on the thermal resistance of the silica layer as the heat diffuses toward the substrate. ${ }^{30}$ Our first step in data treatment is to adjust this background with a spline and to subtract it, which leads to the useful data shown in Fig. 2(a). The signal at short time delays is shown enlarged in Fig. 2(b). It is a superposition of the Brillouin signal from silica, of period $T_{2}$, with the local ringing of the $\mathrm{Al}$ film. The period of the latter is $2 z_{1} / v_{1}$, about 3 ps in the present example. From this an estimate of the Al-film thickness can be extracted, in fair agreement with independent evidence discussed in Sec. V. The onset of the ringing corresponds to the temporal coincidence of probe and pump which is taken as the origin of time delays in Fig. 2(a).

The oscillations of period $T_{2}$ and amplitude $A_{2}$ are then extracted by a fit to an expression of the type

$$
C_{2}+A_{2} \cos \left(2 \pi t / T_{2}-2 \varphi_{2}\right)
$$

The result is shown in red in Fig. 2(a). At times $t \geqslant t_{2}$ the acoustic pulse has reached the silicon substrate. It produces then the total signal shown by the black line, which is adjusted to the sum of two components rather similar to (4). The reflected acoustic pulse gives a signal of same period $T_{2}$ and reduced amplitude $A_{2}^{\prime}$, also shown in red in Fig. 2(a). The transmitted part produces a decaying oscillation of shorter period $T_{3}$ and initial amplitude $A_{3}$ as illustrated separately in Fig. 2(c). It decays owing to the optical absorption of the probe in the substrate, following (3). The amplitude $A_{3}$ is extrapolated to the time $t_{2}$ where the acoustic pulse reaches the interface $\{2-3\}$, as shown in Fig. 2(c). 


\section{THE ACOUSTIC PERTURBATION AND ITS REFLECTION COEFFICIENT}

To obtain a correct expression for the sensitivity ratio, one must know how the acoustic perturbation in media $\{2\}$ and $\{3\}$ reflects the probe light. This depends on the perturbation shape, as will now be described. The acoustic pulse is produced by the initial heating of the metallic film by the pump pulse which to this effect can be viewed as a $\delta$ function at time $t=0$. From each slice $d z$ of the metal film, elementary compressive stress waves are launched in both the positive and negative $z$ directions. As a stress wave in the positive direction reaches the dielectric film $\{2\}$, it is partly transmitted, launching a compressive (negative) strain wave in the film, pictured in Fig. 3(a). The stress wave in the metal that propagates initially in the negative $z$ direction reaches the free surface $\{0-1\}$, where it is totally reflected with a change of sign to become a tensile (positive) stress wave. The latter reaches the interface $\{1-2\}$ after the flight time $\tau=z_{1} / v_{1}$. This produces a zigzag strain pulse in the dielectric, of spatial extent $2 d=2 v_{2} \tau$. Provided the stress reflection at $\{0-1\}$ is perfect, this first zigzag is strictly antisymmetric. $^{25,31}$ Its profile is affected by the evolution with depth of the pump intensity owing to its absorption by the metal film. However, the rapid diffusion of hot electrons homogenizes to a great extent the heating of the metallic film. ${ }^{32}$ This was taken into account in the drawing of the realistic profiles in Fig. 3(a). There are also further zigzags in the train of pulses, as shown in Fig. 3(a). These originate from the successive reflections of the stress waves in the metal at the interface $\{1-2\}$. They reach the dielectric after one to several round trips in the metallic film, that is, at times that are multiples of $2 \tau$. Each reflection attenuates the amplitude by a factor $r_{\mathrm{ac}}$, the acoustic amplitude-reflection coefficient at the metal-dielectric interface $\{1-2\}$. In the case of $\mathrm{Al}$ on silica, $r_{\mathrm{ac}}$ should be small, $r_{\mathrm{ac}} \sim 0.1$ to 0.2 , so that the amplitude of the successive zigzags rapidly decreases. Via the strain-optic effect, the strain produces in $\{2\}$ a refractive index modulation $\delta n(z, t)$.

The strain pulse in $\{2\}$ is a wave-packet of phonons with a rich spectral content, as illustrated in Fig. 3(b). This spectral content is sensitive to the structure of the $\mathrm{Al}$ film and to the nature of the $\mathrm{Al}$-air and the Al-silica interfaces. ${ }^{33}$ The presentation of Fig. 3(b) emphasizes that the spectral content is, for example, strongly dependent on the Al-film thickness. It also depends on various other factors, such as the homogenization in the heating of the metal by hot electrons or the strength of the tail produced by multiple acoustic reflections within the Al film. Each frequency component propagates with nearly
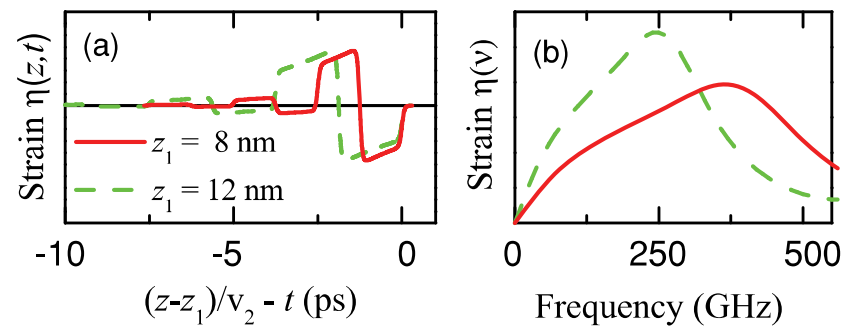

FIG. 3. (Color online) (a) Typical strain pulses in $\{2\}$ and (b) their Fourier analysis. the same phase velocity $v_{2}$, an approximation which is valid within the frequency range of interest in these experiments. However, the higher frequencies experience a significantly higher acoustic attenuation. As a result, the propagating acoustic pulse maintains its antisymmetric property, but its time dependence changes with depth. This has already been used for measuring hypersound attenuation based on the observation of modifications in the echo profile. ${ }^{24}$ It allowed accessing frequencies well above the Brillouin frequency. As explained in the previous section, the modification of spectral content of the acoustic pulse reaching the substrate is also the basis for the attenuation measurements in the present case.

An expression is now developed that describes the reflection of the probe on the index perturbation $\delta n(z, t)$. One should remark that the probe is reflected by the interface $\{2-3\}$ and thus that in $\{2\}$ light propagates both in the positive and negative $z$ direction. On the other hand, in the substrate $\{3\}$ the probe is so strongly absorbed that it only propagates toward the positive $z$ direction. The backreflection coefficient for an incoming EM pulse incident on an acoustic perturbation propagating along $z$ in an otherwise homogeneous dielectric medium can be written ${ }^{25}$

$$
\delta r=i k_{0} \int \delta \tilde{n}(z, t) e^{ \pm 2 i \tilde{k} z} d z .
$$

In this expression, $\delta r$ is the amplitude-reflection coefficient, the amplitude and phase of the reflected optical wave are referred to the plane $z=0, k_{0}$ and $\tilde{k}=k+i \alpha$ are the values of the carrier wave vectors in vacuum and in the unperturbed medium, respectively, and $\delta \tilde{n}$ is the change of complex refractive index owing to the acoustic perturbation. The upper sign applies to an EM wave incoming from the left, $\propto \exp (-i \omega t+i \tilde{k} z)$, the lower sign to one coming from the right. The integral extends over the perturbed region. The expression is only correct to first order in $\delta \tilde{n}$.

First consider a nonabsorbing medium such as $\{2\}$ for which $\tilde{k}$ is real, $\tilde{k}=k$. We assume a perturbation of the form

$$
\delta \tilde{n}=\delta n=-g(x)=-\operatorname{sgn}(x) g(|x|),
$$

where $x \equiv z-Z(t)$. This is an antisymmetric oscillation centered on the plane $z=Z$. This form is that of the main perturbation illustrated in Fig. 3(a). The choice of sign reflects the fact that the acoustic perturbation starts with a compression, that is, a negative strain component $\eta$ perpendicular to the film. The shape function $g$ includes as important factor the appropriate projection of the strain-optic coupling tensor $\mathbf{p}^{28}$ Introducing (6) in (5), one obtains

$$
\delta r \cong \pm 2 k_{0} e^{ \pm 2 i k Z} \int_{0}^{d} \sin 2 k x g(x) d x,
$$

where the upper limit of integration $d$ is the extension of the perturbation in the region $z>Z$. Again, the upper signs are for a wave incident from the left, the lower ones for a wave from the right. In the special case where $g(x)$ is a constant $g$ for $0 \leqslant x \leqslant d$, and with $k d \ll 1$, (7) gives

$$
\delta r \cong \pm 2 k_{0} k e^{ \pm 2 i k Z} g d^{2} .
$$

The phase factor just results from the translation from $x=0$ to $z=0$. It is remarkable that at $Z=0$ the amplitude-reflection coefficient is real and that it changes sign with the direction 
of the incoming EM-probe pulse. This sign change directly follows from the asymmetry in (6).

Consider now a train of successive acoustic pulses, each of the form (6), and of decreasing amplitudes proportional to $1, r_{\mathrm{ac}}, r_{\mathrm{ac}}^{2}, \ldots$, where $r_{\mathrm{ac}}$ is the acoustic reflection coefficient at the metal-dielectric interface $\{1-2\}$. The centers of these successive pulses are located at $Z, Z-2 d, Z-4 d$, etc. Such a train is illustrated in Fig. 3(a). Adding the reflections produced by each of these pulses, keeping the proper phases, one obtains for an EM-wave incident from the left

$$
\begin{aligned}
\delta r \cong & 2 k_{0} e^{2 i k Z}\left[1+r_{\mathrm{ac}} e^{-4 i k d}+r_{\mathrm{ac}}^{2} e^{-8 i k d}+\cdots\right] \\
& \times \int_{0}^{d} \sin 2 k x g(x) d x .
\end{aligned}
$$

Defining $\varsigma^{ \pm} \equiv\left(1-r_{\mathrm{ac}} e^{\mp 4 i k d}\right)^{-1}$, the factor within square brackets is simply $\varsigma^{+}$. The final result, for both propagation directions, can be written in the compact form

$$
\delta r \cong \pm e^{ \pm 2 i k Z} I^{ \pm}
$$

with

$$
I^{ \pm} \equiv 2 k_{0} \varsigma^{ \pm} \int_{0}^{d} \sin 2 k x g(x) d x,
$$

where the upper signs apply to an EM-wave incident from the left and the lower ones to a wave from the right. Again, one should remember that $I^{ \pm}$includes the strain-optic coupling coefficient. While the single pulse result (7) is simply antisymmetric with respect to the direction of the EM-wave propagation, this is not true for the multiple-pulse case owing to the factors $\varsigma^{ \pm}$. However, with $r_{\text {ac }}$ real, besides a multiplicative constant amplitude, these factors simply introduce phase shifts of opposite signs for the two propagation directions.

Now consider the case where $\tilde{k}$ is real but the medium partly absorbs the acoustic waves. This is relevant here. The index perturbation can be written as a superposition of propagating acoustic waves of frequency $\omega_{\mathrm{ac}}$ and wave vector $\tilde{k}_{\mathrm{ac}}$,

$$
\delta n(z, t)=\frac{1}{2 \pi} \int_{-\infty}^{+\infty} \mathcal{N}\left(k_{\mathrm{ac}}\right) e^{-i \omega_{\mathrm{ac}} t+i \tilde{k}_{\mathrm{ac}} z} d k_{\mathrm{ac}} .
$$

Here $\tilde{k}_{\mathrm{ac}}=k_{\mathrm{ac}}+i \alpha_{\mathrm{ac}}$, where the attenuation coefficient $\alpha_{\mathrm{ac}}$ depends on $k_{\mathrm{ac}}$, with $\alpha_{\mathrm{ac}} \ll k_{\mathrm{ac}}$. This is introduced in (5). One uses $v t=Z$, which leads to $\omega_{\mathrm{ac}} t=k_{\mathrm{ac}} Z$, and $\alpha_{\mathrm{ac}} z$ is replaced with $\alpha_{\mathrm{ac}} Z$ owing to the smallness of $\alpha_{\mathrm{ac}}$. The integration in $d z$ then leads to a $\delta$ function, $\delta\left(k_{\mathrm{ac}} \pm 2 k\right)$. The integration in $k_{\mathrm{ac}}$ gives

$$
\delta r=i k_{0} \mathcal{N}(\mp 2 k) e^{ \pm 2 i k Z} e^{-\alpha_{\mathrm{ac}}(2 k) Z} .
$$

This is identical to (10) and (11) except for the attenuation factor $\exp \left[-\alpha_{\mathrm{ac}}(2 k) Z\right]$. In the actual experiment, the relevant value of $k$ changes upon passing from $\{2\}$ to $\{3\}$. While the damping $\alpha_{\mathrm{ac}}\left(2 k_{2}\right)$ is negligible, the factor $\exp \left[-\alpha_{\mathrm{ac}}\left(2 k_{3}\right) L\right]$ can be measured by changing the thickness $L$ of medium $\{2\}$.

Finally, the effect of a complex $\tilde{k}_{3}$ is simply to attenuate the signal by the decaying exponential contained in $\exp \left[2 i \tilde{k}_{3}(Z-\right.$ $\left.\left.z_{2}\right)\right]$, that is by $\exp \left[-4 \pi \kappa_{3}\left(Z-z_{2}\right) / \lambda_{0}\right]$. This leads to the attenuation time constant $\tau_{3}$ given in Eq. (3).

\section{ACCOUNTING FOR MULTIPLE INTERFERENCES}

In explaining the measurement principle, Sec. II did not dwell on difficulties related to multiple interference effects. The latter are fairly complex and very important. Both the probe and the light reflected by the moving strain pulse are multiply reflected by all sample interfaces. This is a technical point for which a general solution in terms of Green functions is available. ${ }^{34}$ However, our particular geometry being sufficiently simple, it is of advantage to obtain closedform expressions. This is developed in Sec. IV A. In Sec. IV B, the results of that analysis are tested by checking the effects of the probe-carrier wavelength on the observed ratio $A_{3} / A_{2}$. This turns out to be a telling exercise.

\section{A. The sensitivity ratio}

Multiple interferences lead to amplitude transmission and reflection coefficients that are simply calculated from Fresnel's equations. We designate by $\tilde{k}_{j}$ the complex carrier wave-vector in medium $\{j\}, \tilde{k}_{j}=\tilde{n}_{j} k_{0}$. The complex refractive index is written $\tilde{n}_{j}=n_{j}+i \kappa_{j}$ and we use for the electric-field wave propagating in the positive $z$ direction the notation $\mathcal{E}^{+} \exp [-i(\omega t-\tilde{k} z)]$. The relevant reflection and transmission coefficients, $r_{i j}$ and $t_{i j}$ respectively, are listed for convenience in Sec. A of the Appendix. That list includes the reflection coefficient $r_{03}$ which relates at $z=0^{-}$the incident probe-field amplitude, $\mathcal{E}_{0}^{+}$, to the reflected amplitude, $\mathcal{E}_{0}^{-}=r_{03} \mathcal{E}_{0}^{+}$, owing to the entire layer structure from $\{1\}$ to $\{3\}$. The field amplitude $\mathcal{E}_{0}^{-}$is, of course, the one that interferes with the probe reflections on the traveling strain pulse, producing the signals pictured in Fig. 2.

To calculate the EM-reflection from the strain pulse, it is necessary to know the probe amplitudes in media $\{2\}$ and $\{3\}$. In $\{2\}$, the probe generates two traveling waves, one to the right $\mathcal{E}_{2}^{+} \exp \left[-i\left(\omega t-k_{2} z\right)\right]$ and one to the left $\mathcal{E}_{2}^{-} \exp \left[-i\left(\omega t+k_{2} z\right)\right]$. In $\{3\}$, there is only a wave to the right, $\mathcal{E}_{3}^{+} \exp \left[-i\left(\omega t-\tilde{k}_{3} z\right)\right]$. It is a standard exercise to obtain the amplitudes $\mathcal{E}_{2}^{+}, \mathcal{E}_{2}^{-}$, and $\mathcal{E}_{3}^{+}$using Maxwell's equations and the boundary conditions. The results are found in Sec. B of the Appendix. These fields are the ones that are reflected from the strain according to (10). The situation is sketched in Fig. 4. In the first time span, $t<t_{2}$, the acoustic wave moves to the right in $\{2\}$ as shown in Fig. 4(a). The wave of amplitude $\mathcal{E}_{2}^{+}$is reflected from the back (B) of the strain pulse, its tail side. The wave of amplitude $\mathcal{E}_{2}^{-}$is reflected from the front $(\mathrm{F})$ side of the (a)

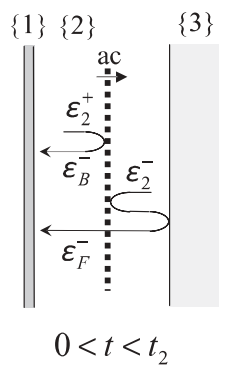

(b)

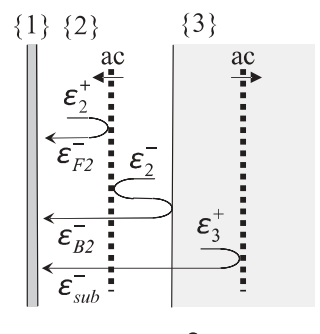

FIG. 4. Sketch of the various EM reflections from the acoustic pulse. 
pulse. The amplitude at $z=z_{1}^{+}$of these reflections is written $\mathcal{E}_{\mathrm{B}}^{-}$and $\mathcal{E}_{\mathrm{F}}^{-}$, respectively. According to $(10), \mathcal{E}_{\mathrm{B}}^{-}$is associated with $+I^{+}$while $\mathcal{E}_{\mathrm{F}}^{-}$with $-I^{-}$. In the second time span, $t>t_{2}$ but below two transit times, the situation is that sketched in Fig. 4(b). For the reflected EM waves, the picture is similar to Fig. 4(a), except that the roles of B and $\mathrm{F}$ are reversed. In the notation $\mathcal{E}_{\mathrm{F} 2}^{-}$and $\mathcal{E}_{\mathrm{B} 2}^{-}$the subscript 2 refers to the second time span. However, the reflected acoustic pulse travels now with its tail at $z>Z$. This reverses the roles of $I^{+}$and $I^{-}$in Eq. (10). Thus, $\mathcal{E}_{\mathrm{B} 2}^{-}$is associated with $-I^{+}$and $\mathcal{E}_{\mathrm{F} 2}^{-}$with $+I^{-}$. The detailed expressions for $\mathcal{E}_{\mathrm{B}}^{-}, \mathcal{E}_{\mathrm{F}}^{-}, \mathcal{E}_{\mathrm{F} 2}^{-}$, and $\mathcal{E}_{\mathrm{B} 2}^{-}$are given in Eqs. (A12)-(A15), respectively. Finally, in medium $\{3\}$, there is only $\mathcal{E}_{3}^{+}$which leads at $z=z_{1}^{+}$to the amplitude noted $\mathcal{E}_{\text {sub }}^{-}$in Fig. 4(b) and Eq. (A16). That amplitude is now proportional to $I_{\text {sub }}^{+}$, which differs strongly from $I^{+}$since it refers to medium $\{3\}$. From $z=z_{1}^{+}$, the five amplitudes are multiply reflected within the structure and finally appear at $z=0^{-}$, where they interfere with $\mathcal{E}_{0}^{-}$. This multiplies all amplitudes by a common coefficient $M$ that only depends on Fresnel-like coefficients and on the phase delay in $\{2\}$,

$$
\Phi_{2}=2 \pi L n_{2} / \lambda_{0}
$$

The value of $M$ is given in Eq. (A17).

We now calculate the coefficients $A_{2}, A_{2}^{\prime}$, and $A_{3}$. During the first time span, $0<t<L / v_{2}$, the delay can be expressed in terms of $\varphi=k_{2}\left(Z-z_{1}\right)$. The reflected probe intensity can be written

$$
\mathcal{F}_{2}(\varphi) \propto \int\left|\mathcal{E}_{0}^{-}+\mathcal{E}_{\mathrm{B}}^{-}+\mathcal{E}_{\mathrm{F}}^{-}\right|^{2} d t \cong C_{2}+A_{2} \cos 2\left(\varphi-\varphi_{2}\right)
$$

where the fields are all at $z=0^{-}$, the integral is over the probe-pulse length, and $A_{2}, C_{2}$, and $\varphi_{2}$ are constants that are determined experimentally. The right-hand side of (15) assumes that $\mathcal{E}_{0}^{-}$is large compared to the other amplitudes so that $C_{2} \gg A_{2}$, which is verified experimentally. For the relevant amplitude $A_{2}$ one finds

$$
A_{2} \propto f_{2}\left|I^{+}\right|,
$$

with the definition of a sensitivity function

$$
f_{2} \equiv\left|M r_{03}^{*}-M^{*} r_{03}\left(r_{23}^{*}\right)^{2} e^{-4 i \phi_{2}}\right| .
$$

It is because $\varsigma^{-}$is the complex conjugate of $\varsigma^{+}$that $\left|I^{+}\right|$ factors out in (16) and that $f_{2}$ is written in this simple form.

During the second time span, $L / v_{2}<t<2 L / v_{2}$, the slow oscillations, which arise from the sound pulse traveling back in the dielectric, are given by

$$
\mathcal{F}_{2}^{\prime}(\varphi) \propto \int\left|\mathcal{E}_{0}^{-}+\mathcal{E}_{\mathrm{B} 2}^{-}+\mathcal{E}_{\mathrm{F} 2}^{-}\right|^{2} d t \cong C_{2}^{\prime}+A_{2}^{\prime} \cos 2\left(\varphi-\varphi_{2}^{\prime}\right) .
$$

The rapid oscillations decaying in time result from the faster dependence of the complex phase $\varphi_{\text {sub }}=\tilde{k}_{3}\left(Z-z_{2}\right)$. Separating this phase into $\varphi_{\text {sub }}^{\prime}+i \varphi_{\text {sub }}^{\prime \prime}$, they can be written

$\mathcal{F}_{3}(\varphi) \propto \int\left|\mathcal{E}_{0}^{-}+\mathcal{E}_{\text {sub }}^{-}\right|^{2} d t \cong C_{3}+A_{3} \cos 2\left(\varphi_{\text {sub }}^{\prime}-\varphi_{3}\right) e^{-2 \varphi_{\text {sub }}^{\prime \prime}}$.
Like for (15), the constants on the right-hand side of (18) and (19) are determined experimentally.

The functions $\mathcal{F}$ depend on the incident field amplitude $\mathcal{E}_{0}^{+}$. The latter can be renormalized out by considering the ratios $A_{2}^{\prime} / A_{2}$ and $A_{3} / A_{2}$. The former is rather trivial. Using (16) and a similar calculation for $A_{2}^{\prime}$, one finds

$$
A_{2}^{\prime} / A_{2}=\left|r_{\mathrm{ac}}^{\mathrm{sub}}\right| \text {, }
$$

where $r_{\mathrm{ac}}^{\mathrm{sub}}$ is the amplitude reflection coefficient of the acoustic pulse at the interface $\{2-3\}$. The ratio $A_{3} / A_{2}$ plays the central role in the measurement. The value of $A_{3}$ is taken at $Z=z_{2}$. One finds then

$$
A_{3} \propto f_{3}\left|I_{\mathrm{sub}}^{+} t_{\mathrm{ac}}^{\mathrm{sub}}\right| e^{-\alpha_{\mathrm{ac}}\left(2 k_{3}\right) L},
$$

where $t_{\mathrm{ac}}^{\text {sub }}$ is the acoustic amplitude transmission coefficient at $\{2-3\}$. The second sensitivity function is

$$
f_{3} \equiv\left|M r_{03}^{*} t_{23} t_{32}\right|
$$

The proportionality factor in (21) is the same as in (16). It follows that

$$
A_{3} / A_{2}=\left(f_{3} / f_{2}\right)\left|I_{\mathrm{sub}}^{+} / I^{+}\right|\left|t_{\mathrm{ac}}^{\mathrm{sub}}\right| e^{-\alpha_{\mathrm{ac}}\left(2 k_{3}\right) L} .
$$

The ratio

$$
f_{3} / f_{2}=\left|t_{23} t_{32}\right|\left|1-\frac{M^{*} r_{03}}{M r_{03}^{*}}\left(r_{23}^{*}\right)^{2} e^{-4 i \phi_{2}}\right|^{-1}
$$

gives the effect of the multiple reflections on the observed ratio $A_{3} / A_{2}$. It is thus called the sensitivity ratio.

Figure 5 illustrates the variation with the film thickness $L$ of the sensitivity functions $f_{2}, f_{3}$ and of their ratio $f_{3} / f_{2}$, as defined in Eqs. (17), (22), and (24). While $f_{3}$ only depends on the round-trip phase $2 \Phi_{2}$ as defined in Eq. (14), $f_{2}$ contains the difference of two terms that depend on twice this round-trip phase, $4 \Phi_{2}$. This produces the double peaks on $f_{2}$ and the nontrivial shape for the sensitivity ratio $f_{3} / f_{2}$ illustrated in Fig. 5. This ratio modulates $A_{3} / A_{2}$, from which the absolute attenuation $\exp \left[-\alpha_{\mathrm{ac}}\left(\nu_{3}^{\mathrm{ac}}\right) L\right]$ is extracted. It is already quite obvious from Fig. 5, and it will become evident in the

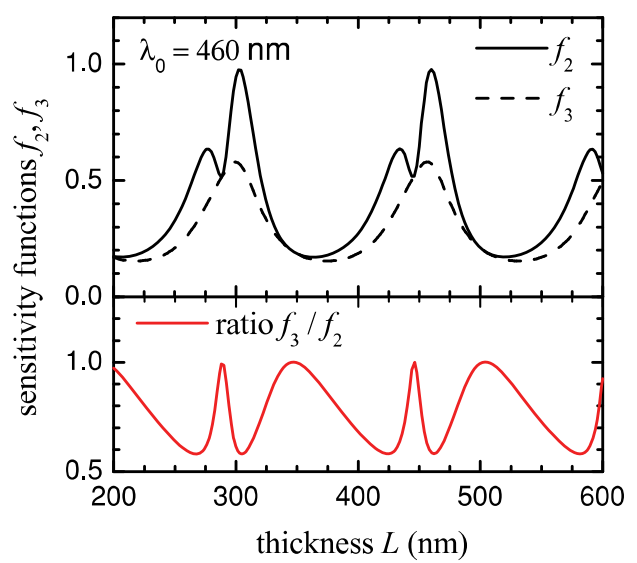

FIG. 5. (Color online) The sensitivity functions $f_{2}$ and $f_{3}$ vs the silica-film thickness $L$. (Bottom) The sensitivity ratio $f_{3} / f_{2}$ which is proportional to the observed signal $A_{3} / A_{2}$. The curves are calculated for an 8 -nm-thick Al film whose refractive index is that of bulk aluminum 


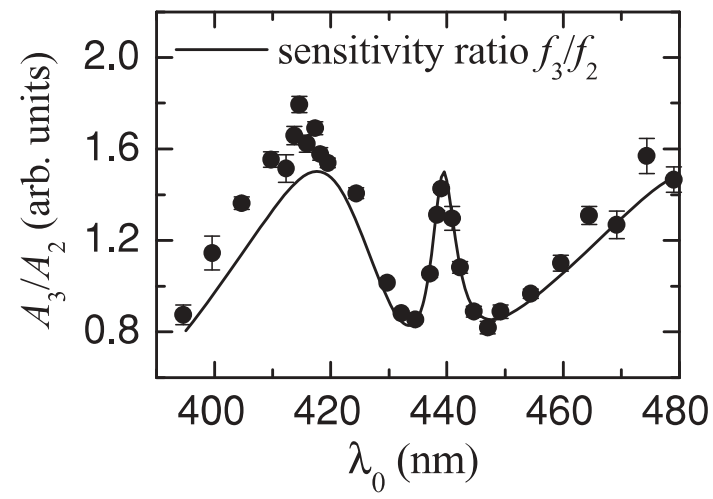

FIG. 6. The measured signals (dots) $A_{3} / A_{2}$ obtained on the wetox sample of $1024 \mathrm{~nm}$ silica thickness as functions of the carrier wavelength $\lambda_{0}$ compared to the shape of the sensitivity ratio.

following sections, that a good knowledge of the time $t_{2}$ and the thicknesses $L$, in addition to all indexes, are strict prerequisites to extract precise attenuation values.

\section{B. Variation with the probe carrier frequency}

To verify the preceding approach, a study of the signal $A_{3} / A_{2}$ as a function of the carrier wavelength $\lambda_{0}$ was performed. The signal is proportional to $f_{3} / f_{2}$ according to (23). The variation of $f_{3} / f_{2}$ with $\lambda_{0}$ is faster for silica films of greater thickness, and thus the experiment in Fig. 6 uses the thickest wetox sample. The shape of the sensitivity ratio illustrated by the line in Fig. 6 is reversed from that in Fig. 5 as the phase $\Phi_{2}$ is proportional to $L$ but inversely proportional to $\lambda_{0}$. The solid line is calculated for the film thicknesses actually derived from the reflection measurements explained in the following section, $L=1024 \mathrm{~nm}$ and $d^{\prime}=8.0 \mathrm{~nm}$, and with the refractive index of bulk $\mathrm{Al} .{ }^{35}$ The experimental values, $A_{3} / A_{2}$, nearly follow the shape of this sensitivity ratio which is obtained without adjustable parameter except for the overall vertical scale. It should be emphasized that the narrow peak in $A_{3} / A_{2}$ at $\lambda_{0} \simeq 440 \mathrm{~nm}$ is remarkably reproduced in $f_{3} / f_{2}$. This provides a strong confirmation for the validity of the analysis. In particular, it supports the antisymmetric form of the main acoustic perturbation shown in Fig. 3(a) as the latter leads to the \pm sign in (10) and thereby to the opposite signs of the fields $\mathcal{E}_{\mathrm{B}}^{-}\left(z_{1}^{+}\right)$and $\mathcal{E}_{\mathrm{F}}^{-}\left(z_{1}^{+}\right)$. It is the latter that add up to finally give the amplitude $A_{2}$ in (15) and thus the minus sign in the expression for $f_{2}$ in (17). A symmetric acoustic perturbation, or a symmetric component as in Ref. 33, would lead to another expression for $f_{2}$, and thus for the sensitivity ratio, in particular for the term in $4 \Phi_{2}$ that leads to the narrow peak in $f_{3} / f_{2}$. Hence, the importance of the check provided in Fig. 6.

However, one should note that a quantitative comparison of the entire dispersion in the signal $A_{3} / A_{2}$ to that in the ratio $f_{3} / f_{2}$ requires taking into account several additional effects. First, the acoustic waves observed in $\mathrm{Si}$ are appreciably attenuated in their transit through the silica layer, as explained in Sec. VI. This attenuation strongly increases for decreasing $\lambda_{0}$, strongly reducing $A_{3}$. Second, the amplitude $A_{3}$ directly depends on the appropriate projection of the strain-optic tensor $\mathbf{p}$ defined by $\delta \epsilon=\mathbf{p} \eta$, where $\delta \epsilon$ is the change in the dielectric constant tensor of silica produced by the acoustic strain $\eta$. The projection $p$ of $\mathbf{p}$ enters $I_{\text {sub }}^{+}$in (21) following $\delta \tilde{n}=\delta \tilde{\epsilon} / 2 \tilde{n}=p \eta / 2 \tilde{n}$. Although $\mathbf{p}$ is not directly available, the related stress-optic tensor $\mathbf{P}$ defined by $\delta \epsilon=\mathbf{P} \sigma$, where $\sigma$ is the stress tensor, has been reported for silicon. ${ }^{36}$ The appropriate projection $p$ is easily determined in terms of $\mathbf{P}$ and the stiffness tensor $\mathbf{C}{ }^{37}$ Its magnitude appreciably increases as $\lambda_{0}$ approaches $390 \mathrm{~nm}$, owing to the direct gap of $\mathrm{Si}$ at $\sim 3.2 \mathrm{eV}$. This compensates in part for the decrease in $A_{3}$ owing to the acoustic absorption. Third, in dividing $A_{3}$ by $A_{2}$ one normalizes the signal produced in the substrate at $v_{3}^{\text {ac }}$ by that from the film at $v_{2}^{\text {ac }}$. That normalization is fine to eliminate the effect of laser-intensity fluctuations in order to compare measurements at fixed $\lambda_{0}$ and various film thicknesses as presented in Sec. VI. However, this normalization depends on the dispersion in the acoustic amplitude ratio at two separate frequencies, obvious from Fig. 3(b). In particular, owing to the rapid increase of $n_{3}$ as $\lambda_{0}$ approaches the direct gap of $\mathrm{Si}$, the frequency $\nu_{3}^{\mathrm{ac}}$ increases then much faster than $v_{2}^{\mathrm{ac}}$, and consequently the acoustic amplitude ratio varies more rapidly with $\lambda_{0}$. This third dispersive effect is the most difficult one to estimate quantitatively as it depends on the frequency content of the initial acoustic pulse and thus on the exact properties of the $\mathrm{Al}$ thin film. Fortunately, provided the $\mathrm{Al}$ films are the same on all samples of a given series, it is not necessary to know the exact frequency content to extract the sound attenuation in the silica films.

\section{REFRACTIVE INDEXES AND SAMPLE THICKNESSES}

This section will show how the optical thicknesses $n_{2} L$ are determined by separate interference measurements. It should first be noted that the measurement of the period $T_{2}$ gives the product $n_{2} v_{2}$ according to (1). On the other hand, the transit time $t_{2}=L / v_{2}$ is well determined by observing the first reflection echo that returns to the front surface at time $2\left(t_{2}+z_{1} / v_{1}\right)$. Finally, the measurement of $n_{2}\left(\lambda_{0}\right) L$ provides a third independent information from which the three quantities $v_{2}, n_{2}$, and $L$ are separately extracted. We observed on the wetox series that $v_{2}$ and $n_{2}$ are fully consistent with their known values for bulk silica. ${ }^{38}$

We also remark that the observation of the decaying oscillations in $\{3\}$, together with Eqs. (2) and (3), makes it possible to determine the dispersion of the complex index $\tilde{n}_{3}$ provided $v_{3}$ is known, which is the case for silicon. The results are shown in Fig. 7. While the real part $n_{3}$ is in excellent agreement with other determinations, we systematically obtain for $\kappa_{3}$ a value which is lower than found in the literature. ${ }^{39}$ We believe that literature values-mostly obtained with measurements based on surface reflection-might be affected by the condition of the surface, whereas our values are more representative of the bulk.

To determine the optical thickness $n_{2} L$, the sample reflections as functions of the probe wavelength $\lambda_{0}$ in absence of pump were measured. The results are illustrated in Fig. 8 for the wetox series. The periodicity of the curves directly reflects the thicknesses. The maximum values depend on the attenuation in the metallic film which is determined by the product $z_{1} \kappa_{1}$. The intensity-reflection coefficient $R_{03}$ is the square modulus of the amplitude-reflection 


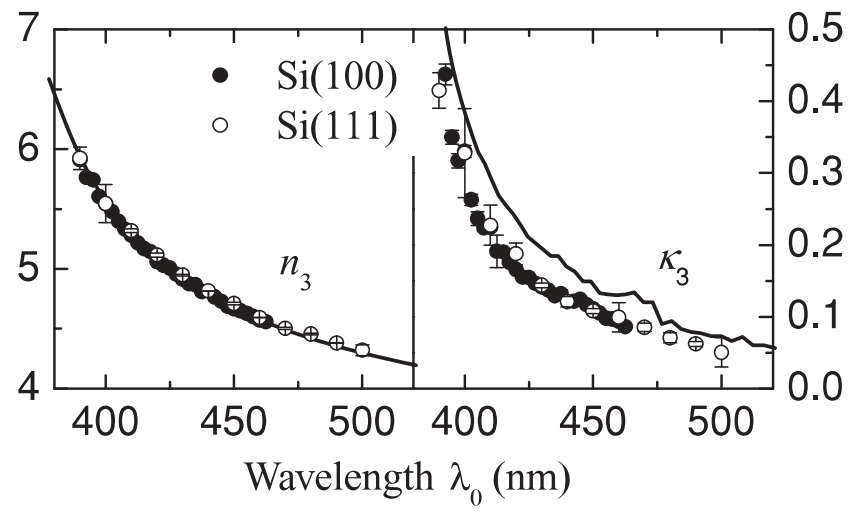

FIG. 7. Dispersion in the refractive index of silicon observed in our measurements. Each data point results from fitting a series of measurements on samples of different silica thicknesses. The points for the (100) and (111) substrate orientations are obtained with silica layers of different origins described in Sec. II. The error bars are smaller for (100) than for (111), owing to the stronger signals in the former case (illustrated in Fig. 9). The solid line is the literature value from Ref. 39.

coefficient given in Eq. (A6), $R_{03}=\left|r_{03}\right|^{2}$. We take the index of silica $n_{2}$ from Ref. 38 and the complex index of silicon $\tilde{n}_{3}$ from the preceding. An indication for the Al-film thickness $z_{1}$ is obtained from the ringing period of the film, $T_{1}=2 z_{1} / v_{1}$. Using the bulk velocity value $v_{1} \simeq 6400 \mathrm{~m} / \mathrm{s}$ and the observed ringing period $T_{1}=3.0 \mathrm{ps}$, one finds $z_{1} \simeq 9.6 \mathrm{~nm}$, which is close to the nominal value of $10 \mathrm{~nm}$. However, applying this together with the bulk value of $\tilde{n}_{1}$ taken from Ref. 35, leads to theoretical values of $R_{03}$ that are too small, pointing to an overestimate of the Al absorption. This suggests that the aluminum might be in part oxidized or also that the film might be somewhat porous. A simultaneous adjustment of all the data in Fig. 8 is then tested using the bulk value of $\tilde{n}_{1}$ and allowing for six adjustable parameters, the five thicknesses $L_{1}$ to $L_{5}$ and an effective Al thickness $z_{1}$. This leads then to extremely good adjustments and to the value $z_{1}=8.1 \pm 0.1 \mathrm{~nm}$. More elaborate effective media models

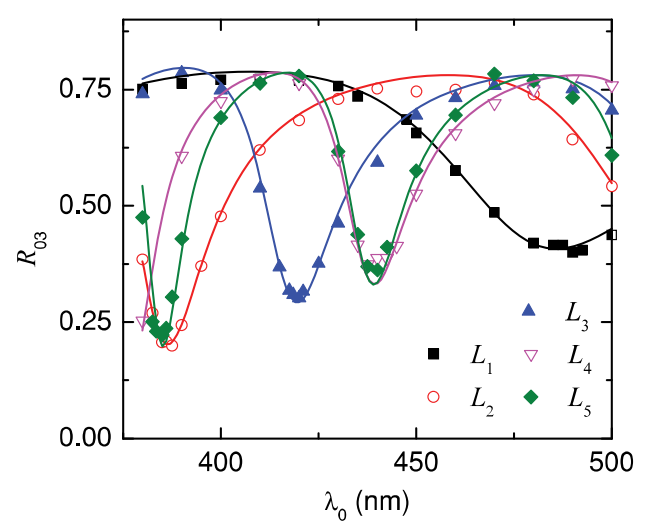

FIG. 8. (Color online) Reflectivity measurements on the five wetox samples as functions of the probe wavelength $\lambda_{0}$. The adjustments explained in the text give $L_{1}=307.8 \pm 0.8, L_{2}=502.6 \pm$ $0.4, L_{3}=691.0 \pm 0.6, L_{4}=874.8 \pm 0.6$, and $z_{1}=8.0 \pm 0.1$, all in $\mathrm{nm}$, with $L_{5}$ fixed at $1023.9 \mathrm{~nm}$. were also tested, taking an effective velocity $v_{1}$ and dielectric constant $\tilde{\epsilon}_{1}$ both depending on a porosity index which itself is taken as the adjustable parameter instead of $z_{1}$. This gives results that are essentially indistinguishable from the preceding ones in terms of $L$ values, but which by construction take into account the observed $T_{1}$. The best value of $L_{5}$ was then used to calculate the sensitivity ratio in Fig. 6 . This revealed that $L_{5}$ needed to be modified by $2 \mathrm{~nm}$ (only $0.2 \%$ ) in order to achieve a perfect match between the measured and calculated peak positions. The simultaneous adjustment of all the data in Fig. 8 was then repeated, however, with only five free parameters, $L_{5}$ being fixed to its best value according to Fig. 6 . These are the final fits shown in Fig. 8.

A similar approach was tested on the LPCVD samples. In that case the period $T_{1}$ is about $4 \mathrm{ps}$, also in fair agreement with the nominal Al thickness of $12 \mathrm{~nm}$. Reflectivity measurements similar to these in Fig. 8 were also performed on these samples. However, a fit with an adjustable $z_{1}$ leads then to the very small value $z_{1}=4.7 \mathrm{~nm}$, almost three times smaller than the nominal thickness. These fits are also poorer, suggesting that the dispersion in the imaginary part $\kappa_{1}$ is not well represented by the bulk index of aluminum. We also had at our disposal the equivalent of Fig. 6 for the LPCVD sample of nominal thickness $1200 \mathrm{~nm}$. It shows a narrow peak similar to that in Fig. 6. However, the thickness derived from the reflectivity curve did not allow matching the peak in $A_{3} / A_{2}$ with that in $f_{3} / f_{2}$. We concluded that the bulk value of $\tilde{n}_{1}$ must be grossly inappropriate when there is such a large difference between the nominal film thickness and the effective one. Given the various results reported in Ref. 40, this is not terribly surprising. At this stage we resorted to adjustments of $A_{3} / A_{2}$ not using the sensitivity ratio for the LPCVD films, as explained in the following section.

\section{ACOUSTIC ATTENUATION RESULTS}

Figure 9 shows two series of room-temperature measurements obtained at a fixed carrier wavelength $\lambda_{0}=430 \mathrm{~nm}$ as functions of the silica thickness $L$. On the left are the wetox films, on the right the LPCVD ones. The data in Fig. 9 are already corrected for the thermal background, as in Fig. 2. A mere inspection shows that for the same values of the oscillation amplitude $A_{2}$, the amplitudes $A_{3}$ decrease with increasing thicknesses $L$. A comparison of both panels also reveals that for the same amplitude $A_{2}$ and at similar thicknesses, for example at $L \simeq 300 \mathrm{~nm}$ - the top traces on both sides-the signal $A_{3}$ is remarkably more intense in the LPCVD films than in the wetox ones. This cannot be ascribed to the different values of the coupling constants $p$ as their estimate following Refs. 36 and 37 shows that $p$ should in fact be appreciably larger for the (111) substrate orientation than for the (100) one. One also notices that the initial ringing of the $\mathrm{Al}$ film is stronger for the wetox films. The difference is also not due to a different acoustic attenuation in the silica films, as these will be found to be quite similar for both types of silica. Owing to the lower velocity $v_{3}$ in the [100] direction, the frequency $v_{3}^{\text {ac }}$ is slightly smaller in that case, but this is also not sufficient to account for the different $A_{3}$ signals. We believe that these differences most probably arise from different transducer properties of the Al films. On the 


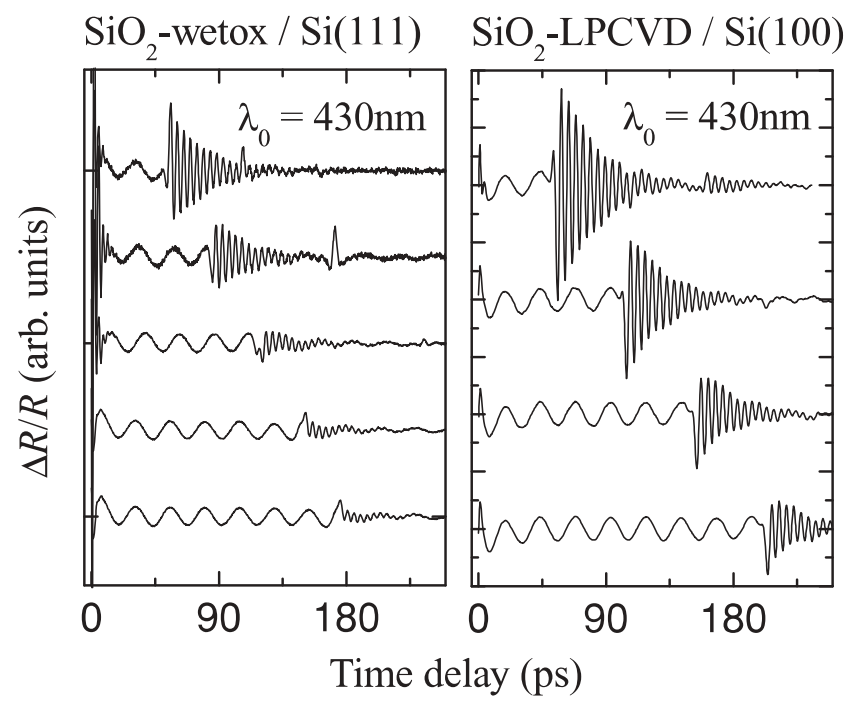

FIG. 9. Two series of measurements at the carrier wavelengths $\lambda_{0}=430 \mathrm{~nm}$ on the wetox samples (left) and on the LPCVD samples (right). From top to bottom, the data are for increasing silica thicknesses, with nominal values of $300,500,700,900$, and $1000 \mathrm{~nm}$ on the left, and nominal values starting at $300 \mathrm{~nm}$ and increasing in steps of $300 \mathrm{~nm}$ on the right.

wetox silica it seems that the metal film rings more strongly but it is less effective in launching the acoustic energy in the silica layer. We also found in the previous section that the optical properties of the $\mathrm{Al}$ film on the wetox samples are remarkably close to these of bulk aluminum, whereas for the $\mathrm{Al}$ film on the LPCVD samples they appear to depart appreciably from bulk values. We are not at this stage in the position to explain the origin of these differences. However, the Al films having been deposited simultaneously on all samples of a given series, and to the extent that these transducers are identical, the preceding aspects should not affect the actual measurement of the variation of $A_{3} / A_{2}$ as a function of $L$ on a given series.

Figure 10 illustrates for the wetox samples typical measured ratios $A_{3} / A_{2}$ as functions of the measured silica thickness $L$. The two panels correspond to two different values of the carrier wavelength $\lambda_{0}$. The continuous curves are the sensitivity ratios $f_{3} / f_{2}$ multiplied by a decreasing exponential and scaled by a constant, $b\left(f_{3} / f_{2}\right) \exp (-\alpha L)$, where $b$ and $\alpha$ are the two free parameters obtained by adjustment of the curves to the five data points. To the extent that there is no appreciable acoustic absorption at the frequency $\nu_{2}^{\text {ac }}$ given by (2), the parameters $\alpha$ extracted in this manner are just the amplitude absorption coefficients $\alpha_{\mathrm{ac}}\left(2 k_{3}\right)$ of (21). The corresponding acoustic frequencies are derived from the periods $T_{3}$ observed in the measurement, $v_{3}^{\mathrm{ac}}$ being given by (2). The energy mean free path $\ell$ relates to $\alpha$ by $\ell^{-1}=2 \alpha$, and the full linewidth that would be obtained in a scattering experiment is then given by $\Gamma / 2 \pi=v_{2} / 2 \pi \ell=\alpha v_{2} / \pi$ in $\mathrm{Hz}$, where $v_{2}\left(\nu_{3}^{\mathrm{ac}}\right)$ is the acoustic velocity at the frequency $v_{3}^{\text {ac }}$, a velocity not measured in this experiment. It is this linewidth value that will be used in the following. It is obvious from the two examples shown in Fig. 10 that it is absolutely necessary to have a very good knowledge of both the sensitivity ratio

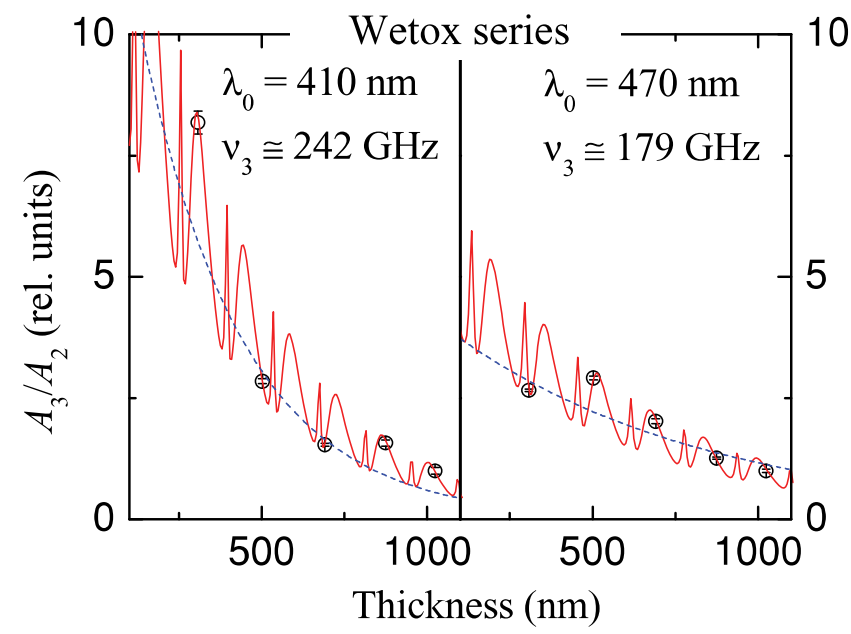

FIG. 10. (Color online) Typical determination of the sound attenuation in the wetox series at two carrier wavelengths $\lambda_{0}$. The dots are the measured values of $A_{3} / A_{2}$ and the lines are fits to $f_{3} / f_{2}$ multiplied by a decaying exponential. The data points do not follow a simple exponential decay owing to the effect of the sensitivity ratio. The linewidths extracted from these fits are $\Gamma / 2 \pi=5.38 \pm 0.11 \mathrm{GHz}$ (left) and $3.43 \pm 0.08 \mathrm{GHz}$ (right). The dotted lines illustrate direct fits of $A_{3} / A_{2}$ to decaying exponentials, with no account for the sensitivity ratio. These rather poor fits give then $\Gamma / 2 \pi=6.2 \pm 2.1$ and $2.5 \pm 0.8 \mathrm{GHz}$, respectively.

$f_{3} / f_{2}$ and the thicknesses $L$ to obtain precise and accurate values for the attenuation constant $\alpha$. Fitting a series of ratios $A_{3} / A_{2}$ simply to decaying exponentials rather than to the correct expression produces random systematic errors, as also illustrated in Fig. 10. The real errors are then unrelated to the error bars of these individual fits.

Varying $\lambda_{0}$, the frequency $v_{3}^{\mathrm{ac}} \equiv \Omega / 2 \pi$ can be changed from $\sim 150$ to slightly above $300 \mathrm{GHz}$. A series of 13 such determinations on the wetox samples is shown in Fig. 11. The values $\Gamma / 2 \pi$ are derived from the corresponding $\alpha$ with a constant $v_{2}=5995 \mathrm{~m} / \mathrm{s}$. All points remarkably align within their small statistical error bars. The same figure shows BLS values obtained in the visible region of the spectrum at three scattering angles. ${ }^{41}$ UV-BLS results are also included, measured at two angles ${ }^{17}$ or at two wavelengths. ${ }^{16}$ The various lines show the theoretical expectations based on Ref. 8 . Up to $\sim 300 \mathrm{GHz}$, there are two main contributions. One is the thermal activation of defects, leading to the dashed curve marked $\Gamma_{\mathrm{TAR}}$ in Fig. 11. The second, which becomes dominant at higher frequencies, is the anharmonic interaction of hypersound with the thermal bath. This leads to $\Gamma_{\text {anh }} \propto \Omega^{2}$. This square law applies up to $\sim 250 \mathrm{GHz}$, with a progressive saturation at higher frequencies, as shown by the dash-dotted line. ${ }^{42}$ The sum $\Gamma_{\mathrm{TAR}}+\Gamma_{\text {anh }}$ agrees remarkably well with the various independent experiments, including the POT determination. One data point at $\sim 1 \mathrm{THz}$ falls well above that sum. It is the lowest frequency point available from IXS at room temperature. ${ }^{43}$ The current understanding is that a third damping mechanism becomes rapidly effective at very high frequencies $\Omega$. It can be attributed to the interaction of hypersound with quasilocal vibrations (QLV) that are also responsible for the emergence of the boson peak (BP). This 


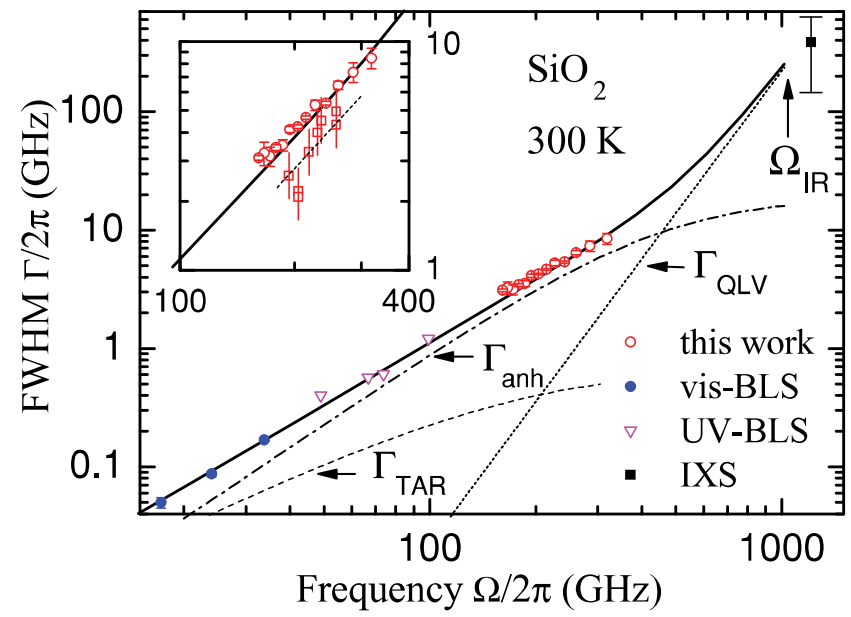

FIG. 11. (Color online) Acoustic linewidths $\Gamma / 2 \pi$ of the wetox samples (this work) compared to visible BLS results (Ref. 41) and UV-BLS ones (Refs. 16 and 17). The point above the Ioffe-Regel frequency $\Omega_{\mathrm{IR}} / 2 \pi$ is from Ref. 43 . The various lines are explained in the text. The inset shows on an expanded scale the same wetox results together with re-evaluated LPCVD ones; the dashed line is a guide to the eye.

leads to a contribution $\Gamma_{\mathrm{QLV}}=B \Omega \Omega^{4} .{ }^{13}$ Such a law has been well observed in cases where the BP falls at sufficiently high frequencies for the region below the $\mathrm{BP}$ to be accessible to IXS. ${ }^{9-12}$ So far, this was not feasible for silica at room temperature. Therefore, there is no experimental point in Fig. 11 above our POT data and up to $\sim 1000 \mathrm{GHz}$. The line marked $\Gamma_{\mathrm{QLV}}$ just shows the expected position of the $\Omega^{4}$ contribution. The solid line is the sum $\Gamma_{\mathrm{TAR}}+\Gamma_{\mathrm{anh}}+\Gamma_{\mathrm{QLV}}$. It agrees rather well with all the data points shown in Fig. 11, as seen enlarged in the inset. The anomalous linewidth increase observed in the inelastic UV scattering (IUVS) experiments ${ }^{18}$ is not included in this graph. That IUVS linewidth matches the solid line up to $\sim 100 \mathrm{GHz}$. Above that value it exhibits a rapid increase that was interpreted as evidence for an acoustic crossover associated with elastic-constant disorder. ${ }^{18}$ If that interpretation were correct, it would be hard explaining why our POT data follow the solid line at higher frequencies. It should be noted that the rapid onset in the IUVS data ${ }^{18}$ exactly corresponds to the onset of strong absorption of the $\mathrm{UV}$ excitation by silica at the energy of $\sim 8 \mathrm{eV}$. It can be suspected that this strong absorption somehow plays a role in the reported linewidth anomaly, although the trivial explanation that the effect is simply due to the uncertainty in the scattering vector owing to absorption ${ }^{41}$ can be ruled out in this case. $^{18}$

The inset of Fig. 11 shows on an expanded scale the wetox measurements with the same solid line together the LPCVD results. ${ }^{44}$ As explained in Sec. V, we were unable to obtain precise values for either $f_{3} / f_{2}$ or the thicknesses $L$ of the LPCVD samples. In this case, the only fits making sense in the equivalent of Fig. 10 for the LPCVD samples are those using simply a decaying exponential. The random systematic errors that are thereby made dominate the accuracy analysis. These errors can be well estimated by applying a similar analysis to the wetox samples for which the correct values are known. In doing this, we adjusted the data points for only four thicknesses to the decaying exponential, as only four thicknesses are available in the LPCVD case. We find then a root-mean-square error on $\Gamma$ which is one-tenth of a decimal order. These are the error bars drawn on the open squares in the inset. The dashed line is a guide to the eye traced through the points and parallel to the solid line. It suggests that the acoustic attenuation of the LPCVD silica is quite similar albeit slightly lower than that of the wetox one.

\section{SUMMARY, DISCUSSION, AND OUTLOOK}

A main point of this work is that POT is able to provide precise values for the acoustic attenuation at sound frequencies that are well above those reachable with either UV-BLS or IUVS. The second important point is that the values found for the wetox samples of vitreous silica in Fig. 11 are in very satisfactory agreement with expectations. However, a slight difference in curvature between the data points and the solid line can be noticed. The increase of $v_{2}\left(v_{3}^{\mathrm{ac}}\right)$ with $v_{3}^{\mathrm{ac}}$ was not included in the calculation of the experimental values of $\Gamma$, but that effect which is at most $\sim 1 \%$-as seen from $^{45}$ would be much too small to modify the appearance of the data. One can think of several explanations. First, the model for the anharmonic width $\Gamma_{\text {anh }}$ in Ref. 8 is the simplest possible one, as it only uses a single phonon-relaxation time $\tau_{\text {th }}$. A more elaborate model is likely to produce a more extended frequency region over which $\Gamma_{\text {anh }}$ saturates and thus a flatter curve. A second possibility is that the wetox process, which exposes the material to a relatively large amount of water, could generate a larger-than-usual quantity of defects, and thereby a higher TAR contribution. That could easily raise the attenuation at $150 \mathrm{GHz}$ slightly above the solid line in Fig. 11, followed by a less rapid increase at higher frequencies as normal for TAR processes, thereby leading to the observed behavior. It is interesting that these new POT results are so precise that they now allow raising such issues.

The success of the experiment clearly depends on the accurate determination of the sample thicknesses and on the availability of all indexes. That also applies to the front $\mathrm{Al}$ film, the very important metallic transducer. On our wetox samples, we observed that this $\mathrm{Al}$ layer has a refractive index dispersion which is close to that of bulk aluminum. This is a rather lucky circumstance, as many different behaviors have been reported for $\mathrm{Al}$ films. ${ }^{40}$ In general, one may envision that such very thin films might not be optically isotropic, with a dielectric constant in plane that could be different from that normal to the plane. The former is the one of importance to the POT analysis, whereas the latter would also intervene in ellipsometric determinations of the index. Further, the $\mathrm{Al}-\mathrm{SiO}_{2}$ interface is likely to play an important role in the POT experiment, in particular for what the strength of the initial ringing and the frequency content of the acoustic pulse launched into the dielectric are concerned. We believe that such effects could explain to a great extent the different signal amplitudes illustrated on both sides of Fig. 9. We do not yet know how this can be successfully controlled. The silicon substrate is also an important element to the success of the experiment. On the one hand, the large 
strength of the signal $A_{3}$ directly results from the extremely large stress-optic coefficients of $\mathrm{Si}$ in the blue region of the spectrum, ${ }^{36}$ typically 100 times larger than that of silica. ${ }^{28}$ On the other hand, the sizable optical absorption of Si effectively prevents any optical interference with reflections that would return from surfaces beyond the interface $\{2-3\}$, thereby keeping the analysis of Sec. IV and the form of the sensitivity ratio within reasonable complexity. The hope of producing similar results on other substrates, for example in achieving higher products $n_{3} v_{3}$ and thereby higher acoustic frequencies, should take good note of these remarkable advantages of silicon.

The most immediate outlook for future work is to perform the same experiment at lower temperatures. On cooling, the TAR contribution $\Gamma_{\mathrm{TAR}}$ is expected to first increase, and this should be rather easily observable. More difficult will be to go into the region where $\Gamma_{\mathrm{QLV}}$ will become dominant. This would require sub-liquid-nitrogen temperatures and experiments with thicker dielectric layers. The thermal heating associated with the POT technique itself might then become a real experimental challenge, but if so it could presumably lead to an enhanced understanding of phonon-thermalization processes. Such experiments might provide the opportunity of directly observing the onset of the $\Omega^{4}$ power law as a function of frequency and temperature, while so far only one tunnel-junction investigation near $1 \mathrm{~K}$ has reported a high power dependence but no onset. ${ }^{46}$

\section{ACKNOWLEDGMENTS}

The authors thank M. Dewitte, A. Leroy, and A. Fattorini from the Institut d'Électronique, de Microélectronique et de Nanotechnologie, in Lille, for providing them with the samples.

\section{APPENDIX: CALCULATING THE INTERFERENCES}

\section{A. Front surface reflection of the probe pulse}

At the interface $\{i-j\}$ between media $\{i\}$ and $\{j\}$, the standard Fresnel formulas apply. The complex transmission amplitude from $\{i\}$ to $\{j\}, t_{i j}$, and the complex reflection amplitude on $\{j\}$ of a wave impinging from $\{i\}, r_{i j}$, are

$$
t_{i j}=\frac{2 \tilde{n}_{i}}{\tilde{n}_{i}+\tilde{n}_{j}} ; \quad r_{i j}=\frac{\tilde{n}_{i}-\tilde{n}_{j}}{\tilde{n}_{i}+\tilde{n}_{j}} .
$$

It is easy to determine the combined reflection and transmission coefficients between media $\{0\}$ and $\{2\}$ of Fig. 1, the phase being referred to the front surface of the metal film at $z=0^{-}$. We designate the one-way complex phase delay in $\{1\}$ by $\Phi_{1}=\tilde{k}_{1} z_{1}$. One finds

$$
\begin{aligned}
& r_{02}=\frac{r_{01}+r_{12} e^{2 i \Phi_{1}}}{1-r_{12} r_{10} e^{2 i \Phi_{1}}} \\
& r_{20}=\frac{r_{21}+r_{10} e^{2 i \Phi_{1}}}{1-r_{12} r_{10} e^{2 i \Phi_{1}}} \\
& t_{02}=\frac{t_{01} t_{12} e^{i \Phi_{1}}}{1-r_{12} r_{10} e^{2 i \Phi_{1}}} \\
& t_{20}=\frac{t_{21} t_{10} e^{i \Phi_{1}}}{1-r_{12} r_{10} e^{2 i \Phi_{1}}}
\end{aligned}
$$

Using these, one easily writes the total reflection of the probe pulse on the front surface of the sample, $r_{03} \equiv \mathcal{E}_{0}^{-} / \mathcal{E}_{0}^{+}$, where $\mathcal{E}_{0}^{+}$and $\mathcal{E}_{0}^{-}$are the incident and reflected amplitudes, both at $z=0^{-}$. With $k_{2}$ real, we define the phase delay in $\{2\}$ by $\Phi_{2}$ according to (14) and obtain

$$
r_{03}=r_{02}+\frac{t_{02} t_{20} r_{23} e^{2 i \Phi_{2}}}{1-r_{23} r_{20} e^{2 i \Phi_{2}}},
$$

where the phase is referred to $z=0$. In terms of the singleinterface reflection coefficients, one finds then

$$
\mathcal{E}_{0}^{-}=\left[r_{01}+r_{12} e^{2 i \Phi_{1}}+r_{01} r_{12} r_{23} e^{2 i \Phi_{2}}+r_{23} e^{2 i\left(\Phi_{1}+\Phi_{2}\right)}\right] \mathcal{E}_{0}^{+} / D,
$$

with the denominator

$$
D \equiv 1+r_{01} r_{12} e^{2 i \Phi_{1}}+r_{12} r_{23} e^{2 i \Phi_{2}}+r_{01} r_{23} e^{2 i\left(\Phi_{1}+\Phi_{2}\right)} .
$$

\section{B. Amplitude of the probe field within the structure}

Owing to its multiple interferences with the various interfaces, the incident probe field $\mathcal{E}_{0}^{+} \exp \left(-i \omega t+i k_{0} z\right)$ produces in the dielectric layer $\{2\}$ a wave traveling toward the positive $z$ direction whose amplitude coefficient is

$$
\mathcal{E}_{2}^{+}=\frac{t_{01} t_{12}}{D} e^{i\left(\Phi_{1}-k_{2} z_{1}\right)} \mathcal{E}_{0}^{+} .
$$

Similarly, the multiple interferences produce within $\{2\}$ a wave traveling toward the negative $z$ direction, of amplitude

$$
\mathcal{E}_{2}^{-}=r_{23} e^{2 i\left(\Phi_{2}+k_{2} z_{1}\right)} \mathcal{E}_{2}^{+}=\frac{t_{01} t_{12} r_{23}}{D} e^{i\left(\Phi_{1}+2 \Phi_{2}+k_{2} z_{1}\right)} \mathcal{E}_{0}^{+} \text {. }
$$

Finally, in the substrate $\{3\}$, there is a wave propagating in the positive $z$ direction of amplitude

$$
\mathcal{E}_{3}^{+}=t_{23} e^{i\left(\Phi_{2}+k_{2} z_{1}-\tilde{k}_{3} z_{2}\right)} \mathcal{E}_{2}^{+}=\frac{t_{01} t_{12} t_{23}}{D} e^{i\left(\Phi_{1}+\Phi_{2}-\tilde{k}_{3} z_{2}\right)} \mathcal{E}_{0}^{+} .
$$

The $z$ dependences of the waves associated with these amplitudes are $\mathcal{E}_{2}^{+} e^{i k_{2} z}, \mathcal{E}_{2}^{-} e^{-i k_{2} z}$, and $\mathcal{E}_{3}^{+} e^{i \tilde{k}_{3} z}$, respectively.

\section{Scattering of the probe field by the acoustic perturbation}

At times $t$ such that $0<t<L / v_{2}$, the acoustic pulse is traveling from $z_{1}$ to $z_{2}$ in the dielectric film. The EM wave coming from the left is reflected according to (10) and (11) in which the upper sign applies. The reflection travels back to position $z=z_{1}^{+}$at which point its amplitude is

$$
\mathcal{E}_{\mathrm{B}}^{-}\left(z_{1}^{+}\right)=I^{+} e^{2 i \varphi} \mathcal{E}_{2}^{+} e^{i k_{2} z_{1}},
$$

where $\varphi \equiv k_{2}\left(Z-z_{1}\right)$. The subscript B alludes to "back," as the signal is produced by a reflection occurring on the back side of the traveling acoustic pulse as illustrated in Fig. 4(a). Similarly, the wave from the right, of amplitude $\mathcal{E}_{2}^{-}$, is reflected on the acoustic perturbation according to (10) and (11), where now the lower sign applies. This reflection travels toward the interface $\{2-3\}$; it is reflected there with $r_{23}$, and it returns to $z_{1}^{+}$where its value is

$$
\begin{aligned}
\mathcal{E}_{\mathrm{F}}^{-}\left(z_{1}^{+}\right) & =-I^{-} r_{23} e^{-2 i \varphi+2 i \Phi_{2}-i k_{2} z_{1}} \mathcal{E}_{2}^{-} \\
& =-I^{-} r_{23}^{2} e^{-2 i \varphi+4 i \Phi_{2}} \mathcal{E}_{2}^{+} e^{i k_{2} z_{1}}
\end{aligned}
$$


The subscript F refers to "front" as the signal is produced by a reflection occurring on the front side of the traveling pulse as sketched in Fig. 4(a).

At times greater than $L / v_{2}$, but below $2 L / v_{2}$, the acoustic pulse has reached the substrate. It is partly reflected at the $\{2-3\}$ interface and partly transmitted, with amplitude coefficients defined as $r_{\mathrm{ac}}^{\mathrm{sub}}$ and $t_{\mathrm{ac}}^{\mathrm{sub}}$, respectively. The reflected acoustic signal also reflects the probe components $\mathcal{E}_{2}^{+}$and $\mathcal{E}_{2}^{-}$just as previously, except that the roles of $I^{+}$and $I^{-}$are interchanged. This situation is sketched in Fig. 4(b). This leads to fields at $z_{1}^{+}$that can be written

$$
\begin{gathered}
\mathcal{E}_{\mathrm{F} 2}^{-}\left(z_{1}^{+}\right)=I^{-} r_{\mathrm{ac}}^{\mathrm{sub}} e^{2 i \varphi} \mathcal{E}_{2}^{+} e^{i k_{2} z_{1}}, \\
\mathcal{E}_{\mathrm{B} 2}^{-}\left(z_{1}^{+}\right)=-I^{+} r_{\mathrm{ac}}^{\mathrm{sub}} r_{23}^{2} e^{-2 i \varphi+4 i \Phi_{2}} \mathcal{E}_{2}^{+} e^{i k_{2} z_{1}}
\end{gathered}
$$

The subscripts B2 and F2 indicate that these fields are observed in the second time span.

The simultaneous signal from the substrate $\{3\}$ is produced by the probe component whose amplitude is given by (A11).
Its reflection on the acoustic perturbation travels to $z=z_{2}^{+}$, it is transmitted through the $\{3-2\}$ interface, and travels to $z_{1}^{+}$at which point it equals

$$
\mathcal{E}_{\mathrm{sub}}^{-}\left(z_{1}^{+}\right)=I_{\mathrm{sub}}^{+} t_{\mathrm{ac}}^{\mathrm{sub}} t_{23} t_{32} e^{2 i \varphi_{\mathrm{sub}}+2 i \Phi_{2}} \mathcal{E}_{2}^{+} e^{i k_{2} z_{1}},
$$

where $\varphi_{\text {sub }} \equiv \tilde{k}_{3}\left(Z-z_{2}\right)$ is the one-way complex delay in the substrate. The subscript "sub" on $I$ emphasizes that this is now the quantity appropriate to the substrate. $I_{\text {sub }}^{+}$differs from $I^{+}$ by different values of $d$ and $\tilde{k}=\tilde{k}_{3}$, as well as by a different acousto-optic coefficient hidden in the function $g$ of Eq. (6).

The fields (A12) to (A15) are multiply reflected within $\{2-3\}$ and transmitted to $z=0^{-}$with the coefficient $t_{20}$ given in (A5). As a result, the common multiplicative factor $\mathcal{E}_{2}^{+} e^{i k_{2} z_{1}}$ in (A12)-(A16) is replaced with $M \mathcal{E}_{0}^{+}$,

$$
M=\frac{t_{02} t_{20}}{\left(1-r_{23} r_{20} e^{2 i \Phi_{2}}\right)^{2}} .
$$

The field components at $z=0^{-}$interfere with $\mathcal{E}_{0}^{-}=r_{03} \mathcal{E}_{0}^{+}$, leading to the observed interference oscillations.
${ }^{1}$ A. Anderson, B. Halperin, and C. Varma, Philos. Mag. 25, 1 (1972).

${ }^{2}$ W. A. Phillips, J. Low Temp. Phys. 7, 351 (1972).

${ }^{3}$ J. Jäckle, Z. Physik 257, 212 (1972).

${ }^{4}$ J. Jäckle, L. Piché, W. Arnold, and S. Hunklinger, J. Non-Cryst. Solids 20, 365 (1976).

${ }^{5}$ W. A. Phillips, Rep. Prog. Phys. 50, 1657 (1987).

${ }^{6}$ D. Tielbürger, R. Merz, R. Ehrenfels, and S. Hunklinger, Phys. Rev. B 45, 2750 (1992).

${ }^{7}$ R. Vacher, J. Pelous, F. Plicque, and A. Zarembowitch, J. Non-Cryst. Solids 45, 397 (1981).

${ }^{8}$ R. Vacher, E. Courtens, and M. Foret, Phys. Rev. B 72, 214205 (2005).

${ }^{9}$ B. Rufflé, M. Foret, E. Courtens, R. Vacher, and G. Monaco, Phys. Rev. Lett. 90, 095502 (2003).

${ }^{10}$ B. Rufflé, G. Guimbretière, E. Courtens, R. Vacher, and G. Monaco, Phys. Rev. Lett. 96, 045502 (2006).

${ }^{11}$ G. Monaco and V. M. Giordano, Proc. Natl. Acad. Sci. USA 106, 3659 (2009)

${ }^{12}$ G. Baldi, V. M. Giordano, G. Monaco, and B. Ruta, Phys. Rev. Lett. 104, 195501 (2010)

${ }^{13}$ B. Rufflé, D. A. Parshin, E. Courtens, and R. Vacher, Phys. Rev. Lett. 100, 015501 (2008)

${ }^{14}$ W. Schirmacher, Europhys. Lett. 73, 892 (2006).

${ }^{15}$ W. Schirmacher, G. Ruocco, and T. Scopigno, Phys. Rev. Lett. 98, 025501 (2007).

${ }^{16}$ C. Masciovecchio, A. Gessini, S. Di Fonzo, L. Comez, S. C. Santucci, and D. Fioretto, Phys. Rev. Lett. 92, 247401 (2004).

${ }^{17}$ P. Benassi, S. Caponi, R. Eramo, A. Fontana, A. Giugni, M. Nardone, M. Sampoli, and G. Viliani, Phys. Rev. B 71, 172201 (2005).

${ }^{18}$ C. Masciovecchio, G. Baldi, S. Caponi, L. Comez, S. Di Fonzo, D. Fioretto, A. Fontana, A. Gessini, S. C. Santucci, F. Sette, G. Viliani, P. Vilmercati, and G. Ruocco, Phys. Rev. Lett. 97, 035501 (2006).

${ }^{19}$ F. Sette, M. H. Krisch, C. Masciovecchio, G. Ruocco, and G. Monaco, Science 280, 1550 (1998).
${ }^{20}$ C. Thomsen, H. T. Grahn, H. J. Maris, and J. Tauc, Phys. Rev. B 34, 4129 (1986).

${ }^{21}$ B. Bonello, B. Perrin, E. Romatet, and J. C. Jeannet, Ultrasonics 35, 223 (1997).

${ }^{22}$ O. B. Wright, B. Perrin, O. Matsuda, and V. E. Gusev, Phys. Rev. B 64, 081202 (2001).

${ }^{23}$ O. B. Wright, B. Perrin, O. Matsuda, and V. E. Gusev, Phys. Rev. B 78, 024303 (2008).

${ }^{24}$ T. C. Zhu, H. J. Maris, and J. Tauc, Phys. Rev. B 44, 4281 (1991)

${ }^{25}$ H. N. Lin, R. J. Stoner, H. J. Maris, and J. Tauc, J. Appl. Phys. 69 , 3816 (1991).

${ }^{26}$ P. Emery and A. Devos, Appl. Phys. Lett. 89, 191904 (2006).

${ }^{27}$ A. Devos, M. Foret, S. Ayrinhac, P. Emery, and B. Rufflé, Phys. Rev. B 77, 100201 (2008).

${ }^{28}$ A. Devos and R. Côte, Phys. Rev. B 70, 125208 (2004).

${ }^{29}$ B. C. Daly, K. Kang, Y. Wang, and D. G. Cahill, Phys. Rev. B 80, 174112 (2009).

${ }^{30}$ B. C. Daly, H. J. Maris, W. K. Ford, G. A. Antonelli, L. Wong, and E. Andideh, J. Appl. Phys. 92, 6005 (2002).

${ }^{31} \mathrm{C}$. Thomsen, H. T. Grahn, H. J. Maris, and J. Tauc, Opt. Commun 60, 55 (1986)

${ }^{32}$ G. Tas and H. J. Maris, Phys. Rev. B 49, 15046 (1994).

${ }^{33}$ B. C. Daly, T. B. Norris, J. Chen, and J. B. Khurgin, Phys. Rev. B 70, 214307 (2004).

${ }^{34}$ O. Matsuda and O. B. Wright, J. Opt. Soc. Am. B 19, 3028 (2002).

${ }^{35}$ D. Y. Smith, E. Shiles, and M. Inokuti, in Handbook of Optical Constants of Solids, edited by E. D. Palik (Academic Press, New York, 1985), pp. 369-387.

${ }^{36}$ P. Etchegoin, J. Kircher, and M. Cardona, Phys. Rev. B 47, 10292 (1993).

${ }^{37}$ For the (111) substrate orientation one obtains $p=\left[\left(P_{11}+\right.\right.$ $\left.\left.2 P_{12}\right)\left(C_{11}+2 C_{12}\right)-4 P_{44} C_{44}\right] / 3$. For the (100) substrate one finds $p=\left(P_{11}+P_{12}\right) C_{12}+P_{12} C_{11}$

${ }^{38}$ I. H. Malitson, J. Opt. Soc. Am. 55, 1205 (1965).

${ }^{39}$ D. F. Edwards, in Handbook of Optical Constants of Solids, edited by E. D. Palik (Academic Press, New York, 1985), pp. 547-569. 
${ }^{40}$ H. V. Nguyen, I. An, and R. W. Collins, Phys. Rev. B 47, 3947 (1993).

${ }^{41}$ R. Vacher, S. Ayrinhac, M. Foret, B. Rufflé, and E. Courtens, Phys. Rev. B 74, 012203 (2006).

${ }^{42}$ The actual value of $\Gamma_{\text {anh }}$ drawn in Fig. 11 equals 1.2 times that in Ref. 8. This is well within the accuracy of the analysis presented there, which is all based on measurements at frequencies lower than or equal to $35 \mathrm{GHz}$. The current value is selected so that the continuous line coincides with the most accurate BLS data point at $35 \mathrm{GHz}$.
${ }^{43}$ C. Masciovecchio, G. Ruocco, F. Sette, P. Benassi, A. Cunsolo, M. Krisch, V. Mazzacurati, A. Mermet, G. Monaco, and R. Verbeni, Phys. Rev. B 55, 8049 (1997).

${ }^{44}$ The POT data on the LPCVD films shown in Figs. 9 and 11 are the same that were reported in Ref. 27. The linewidths were re-evaluated as explained here.

${ }^{45}$ B. Rufflé, S. Ayrinhac, E. Courtens, R. Vacher, M. Foret, A. Wischnewski, and U. Buchenau, Phys. Rev. Lett. 104, 067402 (2010).

${ }^{46}$ W. Dietsche and H. Kinder, Phys. Rev. Lett. 43, 1413 (1979). 